\title{
Adaptive linear TFPF for seismic random noise attenuation
}

\author{
Juan $\mathrm{Li}^{1} \cdot$ Kexin Meng ${ }^{1} \cdot$ Yuan $\mathrm{Li}^{1} \cdot$ Yue $\mathrm{Li}^{1}$ \\ Received: 5 April 2017 / Accepted: 26 November 2017 / Published online: 22 February 2018 \\ (c) The Author(s) 2018. This article is an open access publication
}

\begin{abstract}
To achieve a high level of seismic random noise suppression, the conventional time-frequency peak filtering (TFPF) has been adequately studied and applied in previous researches in recent years. A window is used to improve linearity, thereby achieving the unbiased estimates. However, applying a fixed window length to all frequencies signals will result in serious loss of effective components. The recently proposed the parabolic-trace TFPF (PT-TFPF) resample seismic record along parabolic-trace to enhance linearity. But complex events in field data have different curvatures, and it is difficult to fit parabolic-trace. To resolve these problems, we present an adaptive linear TFPF (AL-TFPF) in this paper. In this novel method, the linearity of the effective signals is implemented by grouping instead of window or filtering trace. Based on temporal continuity and spatial correlation, grouping is able to adaptively resample. The AL-TFPF stores the similar blocks into a new matrix. In this approach, degree of improvement of linearity is related to the accuracy of block matching. Finally, we evaluate the performance of our method on both synthetic records and field data. The experimental results illustrate that our proposed method realizes the retention of useful components and attenuation of the noise simultaneously, compared with the conventional TFPF and the PT-TFPF.
\end{abstract}

Keywords Seismic signal · Block matching · Time-frequency peak filtering (TFPF) $\cdot$ Random noise suppression

\section{Introduction}

The seismic signals describing the underlying geological structure are usually polluted by random noise during the data collection. The more complex geological structure, the lower SNR in seismic record will be. However, poor performance in a nonstationary circumstance restricts the application in some seismic records, especially in the low signal-to-noise ratio (SNR) case. Thus, the improvement of SNR is the critical step of seismic signal processing. How to effectively attenuate random noise, particularly for the records with strong random noise, is the central issue.

Various noise attenuation methods have been proposed and applied to suppress seismic random noise, such as adaptive filtering (Lin et al. 2015), independent component analysis (Wang et al. 2010), fx-deconvolution (Chen and Ma 2014), and tx-prediction filtering (Abma and claerbout 1995). In recent years, noise attenuation in transform domain

Juan Li

ljuan@jlu.edu.cn

1 College of Communication and Engineering, Jilin University, Changchun 130012, China has become a popular researching trend, such as Fourier transform (Liu and Sacchi 2004), wavelet transform (Liu et al. 2014a, b), Radon transform (Trad et al. 2002), curvelet transform (Górszczyk et al. 2014), and shearlet transform (Liu et al. 2014a, b).

After being proposed, there has been an increasing awareness of time-frequency peak filtering (TFPF) due to its outstanding performance in suppressing nonstationary and strong seismic random noise (Lin et al. 2011). It can effectively recover seismic signal even when it is under the circumstances when the SNR is as low as $-9 \mathrm{~dB}$. However, the fixed window length (WL) leads to a pair of contradictions between random noise reduction and desired signal reservation. The remarkable filtering effectiveness is based on the foundation of linearity unbiased estimation, which results in time-windowing processing in the time domain (Lin et al. 2011).

In order to improve the degree of linearity, some improved TFPF algorithms have been already proposed. In the varying WL TFPF (Lin et al. 2008), the long WL and the short WL are applied to signal segments and noise segments, respectively. But these two kinds of segments are difficult to be distinguished. In the radial-trace TFPF ( $\mathrm{Wu}$ 
et al. 2011), linearity of the effective signals is enhanced by radial-trace transform. However, radial filtering traces with fixed $45^{\circ}$ angle limit its advantages. Parabolic-trace TFPF (PT-TFPF) (Tian and Li 2014) and variable eccentricity hyperbolic-trace TFPF (VH-TFPF) (Tian et al. 2014) have been proposed. They attempt to resample seismic data through the parabolic-trace and hyperbolic-trace. In case that resampling trace fits reflection event perfectly, the events become more clear and continuous by these two methods. However, filtering trace is difficult to match the mixed seismic events perfectly, particularly in field data.

In this article, we propose a novel method-adaptive linear TFPF (AL-TFPF). Firstly, the noisy seismic record is divided into some overlapping fragments. Similar blocks measured by $l^{p}$-norm are stacked together to structure a new matrix that we term it "group." In this processing, temporal continuity and spatial correlation of seismic events are taken into account. Because of matching operation, similarity in grouped fragments enhances linearity greatly. Then, applying TFPF along inter-block traces will improve the results of de-noising. Grouping is an adaptive processing rather than a changeless operation, such as definite length window or some fixed filtering traces. As a conclusion, the method proposed in this article is a perfect fit for the seismic records.

The outline of this paper is as follows. In "Basic principle of the conventional TFPF" section, we present the basic principle and discuss bias of the conventional TFPF. In "Adaptive linear TFPF" section, we describe the algorithm details and implementation of AL-TFPF. In "Application to the seismic records" section, we show the performance of AL-TFPF on both synthetic models and field data and compare with the other methods. Finally, we draw conclusions at the end of the article.

\section{Basic principle of the conventional TFPF}

\section{Principle of TFPF}

The conventional TFPF is an effective method to reconstruct the useful signals from the additive random noise. Assume that noisy signal model is $s(t)=x(t)+n(t)$, where $x(t)$ is pure seismic record and $n(t)$ is additive random noise. In this approach, noisy signal $s(t)$ is encoded into an instantaneous frequency of an analytic signal $Z_{S}(t)$. The peak of the Wigner-Ville spectrum of the analytic signal in the time-frequency domain is the useful components of seismic signal.

Firstly, signal can be modulated, according to the equation:

$Z_{S}(t)=\mathrm{e}^{j 2 \pi \mu \int_{0}^{t} s(\lambda) \mathrm{d} \lambda}$ where $\mu$ is the scaling parameter, which represents the frequency modulation index.

And then, the peak of the time-frequency distribution of $Z_{S}(t)$ is extracted as the filtered signal $\hat{s}(t)$ (Boashash and Mesbah 2004):

$\hat{s}(t)=\frac{\arg _{f} \max \left[W_{Z}(t, f)\right]}{\mu}$

where $W_{Z}(t, f)$ denotes the pseudo Wigner-Ville distribution (WVD) $Z_{S}(t)$ of and is given by

$W_{Z}(t, f)=\int_{-\infty}^{\infty} Z\left(t+\frac{\tau}{2}\right) Z^{*}\left(t-\frac{\tau}{2}\right) \mathrm{e}^{-j 2 \pi f \tau} \mathrm{d} \tau$

\section{Bias analysis of TFPF}

For special cases that the signal is linear in time and signals have been polluted by stationary white Gaussian noise, TFPF could give an absolutely unbiased estimation (Boashash and Mesbah 2004). However, this condition is usually not satisfied. In that way, the bias $B(t)$ of the TFPF algorithm is evaluated as

$$
\begin{aligned}
B(t)= & \arg _{f} \max \left(W_{Z}(t, f) \times \frac{4 \pi^{2} k_{n_{2}} \mu^{2}}{\left(2 \pi^{2} k_{n_{2}} \mu^{2}\right)^{2}+(2 \pi f)^{2}}\right) \cdot \frac{1}{\mu} \\
& -x(t)
\end{aligned}
$$

where $k_{n_{2}}$ is the second cumulant of $n(t)$ (Boashash and Mesbah 2004).

If the TFPF can provide an unbiased estimation of a linear signal $x(t)=\alpha t+\beta$, where $\alpha$ and $\beta$ are constants, $W_{Z}(t, f)$ is

$W_{Z}(t, f)=\delta(f-\mu x(t))$

Derived from this, the bias $B(t)$ becomes

$$
B(t)=\arg _{f} \max \left(\frac{4 \pi^{2} k_{n_{2}} \mu^{2}}{\left(2 \pi^{2} k_{n_{2}} \mu^{2}\right)^{2}+(2 \pi f-2 \pi \mu x(t))^{2}}\right) \cdot \frac{1}{\mu}
$$$$
-x(t)=0
$$

In practice, the conventional TFPF often adopts the pseudo Wigner-Ville distribution (PWVD) (Boashash and Mesbah 2004). It is the windowed version of WVD to satisfy the local linearity and reduce the bias. $h(\tau)$ is a window function sliding with time. The PWVD is defined as

$\mathrm{PW}_{Z}(t, f)=\int_{-\infty}^{\infty} h(\tau) Z\left(t+\frac{\tau}{2}\right) Z^{*}\left(t-\frac{\tau}{2}\right) \mathrm{e}^{-j 2 \pi f \tau} \mathrm{d} \tau$

So, the recovery result using the conventional TFPF is sensitive to window length. An empirical calculation method of window length is presented (Lin et al. 2007).

$1 \leq \mathrm{WL} \leq \frac{0.384 f_{s}}{f_{d}}$ 
where $f_{s}$ is the sampling frequency and $f_{d}$ is the dominant frequency of seismic signal.

However, a fixed WL is not suitable for all frequency components of the signal. The noisy signal contains two events, whose dominant frequencies are 25 and $45 \mathrm{~Hz}$, respectively. The sampling frequency is $1000 \mathrm{~Hz}$ and the offset is $30 \mathrm{~m}$. According to Eq. 8, the maximum optimal WL of $25 \mathrm{~Hz}$ is 15 points and the maximum optimal WL of $45 \mathrm{~Hz}$ is 7 points. The simulation results in Fig. 1 validate the effects of inappropriate window lengths on both signal amplitude and noise suppression. The longer the window, the stronger the noise suppression capability, the more severe the effective signal attenuation; otherwise, the effective signal amplitude is better, but with more random noise residue.

Clearly, different frequency signals correspond to different optimal WLs. Furthermore, dominant frequency of seismic signal as a priori information is unknown in field seismic data.

\section{Adaptive linear TFPF}

To resolve the problems in the conventional TFPF and the PT-TFPF, we propose an AL-TFPF for seismic random noise attenuation in this article. The novel method contains parts of the grouping by matching, inter-block trace TFPF and aggregation.

\section{Grouping by matching}

According to the bias analysis, we know that the linearity of the desired signal has an important influence on the unbiased estimation. Therefore, the conventional TFPF and several

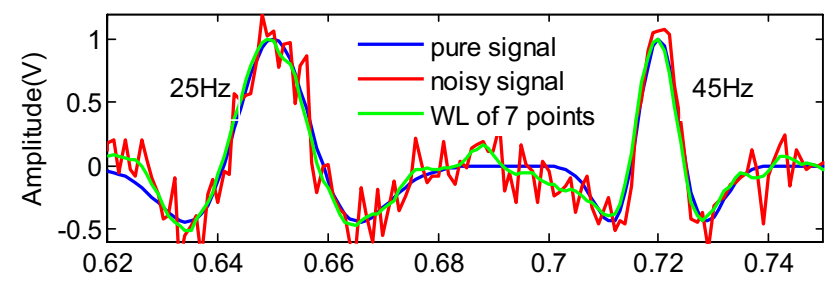

(a)

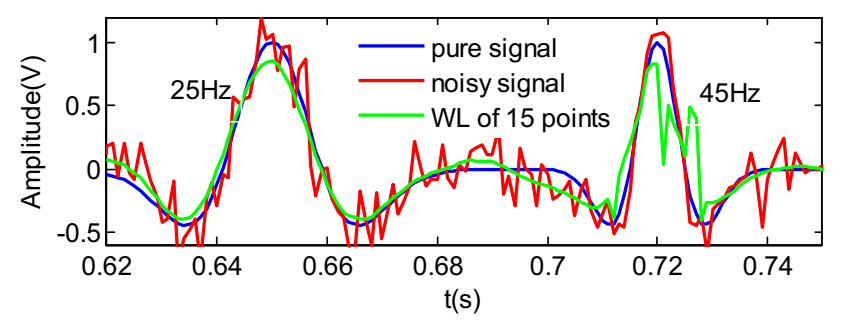

(b)

Fig. 1 a Result of WL of 7 points. b Result of WL of 15 points improved algorithms have done a lot of research in the field of linearity enhancement. The PT-TFPF and the VH-TFPF apply resampling along some filtering traces with fixed shape. Thus, similarity degree between resampling trace and bended reflection events directly influences the noise suppressing effect of improved TFPF algorithm. However, the filtering traces based on some fixed form cannot be applicable for properties (i.e., curvature and inclination) of arbitrary seismic event, in particular for field seismic data containing scattered events.

As previously described, the seismic events are temporal continuous in single channel and spatial correlation between adjacent channels. These properties lead to similarity on waveform of reflected event. However, in the seismic record there is often random noise whose energy is concentrated in certain directions; the noise in these directions is correlated. Based on this, we propose grouping by matching - an adaptive resampling method. We divide each channel into blocks with fixed length $N_{1}$ and the whole data are divided into $N$ blocks. Blocks are overlapped with step $N_{p}$. Ideally, block of noise and block of signal can be effectively divided according to some similarity criteria.

Select a block as the reference block $Y_{R}$ and find candidate blocks $Y_{x}(x \in[1, N])$ which are similar to the given reference one. Figure 2 gives the detailed procedure of the block matching process. Similar blocks we desired are marked by dashed frame. The similarity measure is calculated by weighted Euclidean distance ( $l^{p}$-norm, $p=2$ ), such as

$d\left(Y_{R}, Y_{x}\right)=\frac{\left\|T\left(Y_{R}\right)-T\left(Y_{x}\right)\right\|_{2}^{2}}{N_{1}}$

In order to avoid erroneous grouping caused by large noise variance $\sigma$, we apply discrete wavelet transform $T(\cdot)$ to each block. The wavelet transform divides the signals into low scale and high scale, and the effective signals are mainly distributed on the low scale. So we extract approximate coefficients to calculate distance $d\left(Y_{R}, Y_{x}\right)$. Similar block matching is performed for the simulated seismic record with real

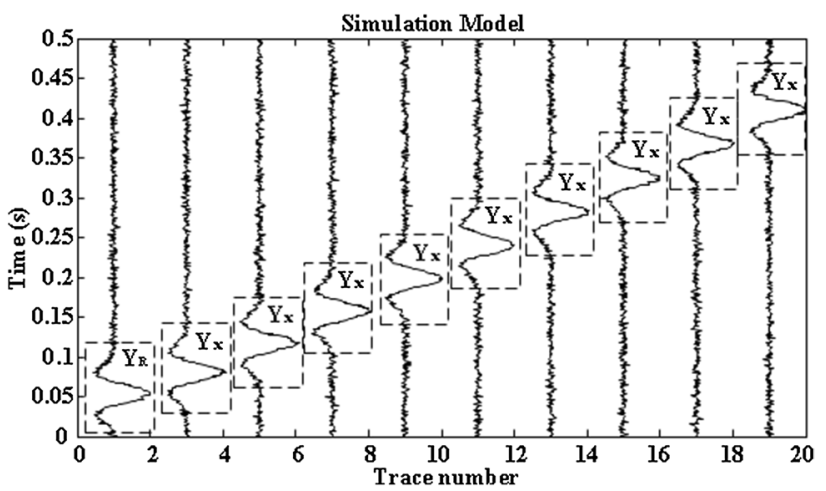

Fig. 2 Diagram of block matching 
noise, in different domains, and the results are shown in Fig. 3. Comparing with matching operation in time domain, we can clearly see that the matching result after wavelet transform is clustered in a smaller area.

The blocks whose distance $d\left(Y_{R}, Y_{x}\right)$ are smaller than a given threshold $\tau$ are considered mutually similar and stacked together as a group $Z_{R}$ belong to $Y_{R}$.

$Z_{R}=\left\{Y_{x}, x \in[1, N] \mid d\left(Y_{R}, Y_{x}\right) \leq \tau\right\}$

Apparently, size of $Z_{R}$ is $N_{1} \times n$, where $n$ is quantity of block similar to $Y_{R}$. Groups belong to noise and noisy signal is shown as Fig. 4. Apparently, if the reference block contains the useful waveform, the blocks in $Z_{R}$ should all contain reflected events.

\section{Inter-block trace TFPF}

In the above approach, to achieve an unbiased result, the desired signal needs to have high linearity. By Eq. 6, we have proved this conclusion that bias of filtering is close to 0 if seismic signal is linear. Obviously, the conventional TFPF that filters along the time direction does not sufficiently take this issue into account.

In our method, grouping by matching is a procedure that transforms seismic record blocks into some new data matrixes whose size is $N_{1} \times n$. Because of the similarity, reflection event and noise are divided into different groups. We term horizontal direction as inter-block trace. There is no doubt that the desired signal is linear for noise group. Moreover, the signal amplitude of corresponding position along inter-block trace should be approximate. As a result, we can infer that the linearity is enhanced, which is compared with time direction. This conclusion is verified in Fig. 5.

Figure 5a exhibits a group divided from a 50-channel noisy synthetic seismic record with one reflection event. Figure $5 \mathrm{~b}$ shows the curve along inter-block trace. Figure $5 \mathrm{c}$ shows a block in this group. That is to say, the line depicted in Fig. $5 \mathrm{c}$ is along the time direction. Through observation and comparison, we can see that the curve extracted along inter-block trace is smoother than time trace. This describes higher degree

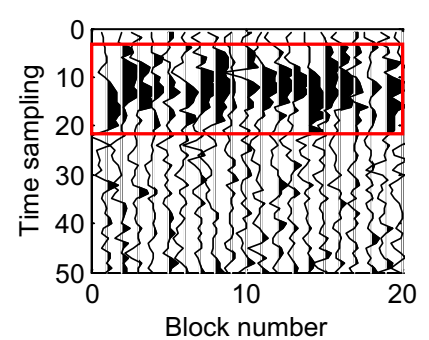

(a)

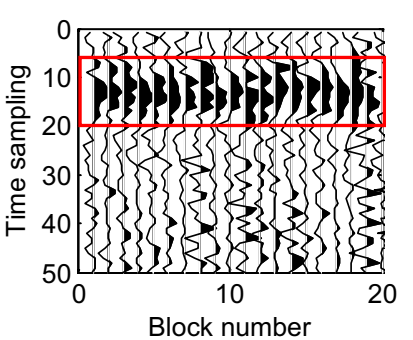

(b)
Fig. 3 Grouping results. a Matching in time domain. b Matching in wavelet domain

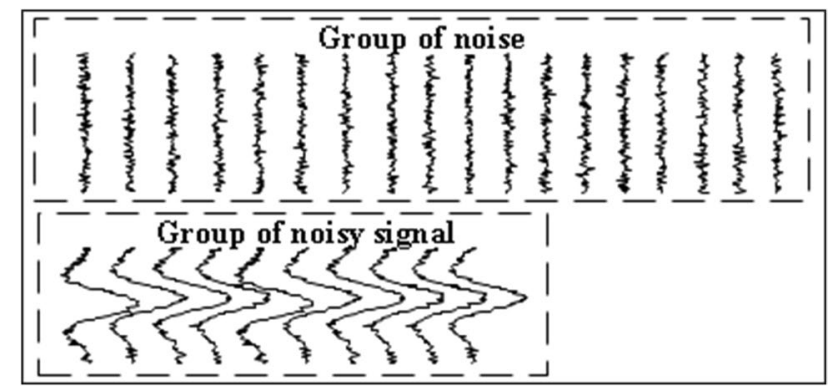

Fig. 4 Diagram of group

of linearity and lower instantaneous frequency which realize lower deviation.

Figure 6 shows de-noising results using two methods, including time trace TFPF $(\mathrm{WL}=13)$ and inter-block trace TFPF in this group. It is easy to deduce that waveform denoised by inter-block trace matches the reflection event in shape better than the one de-noised by time trace. In addition, proposed method does not need any prior knowledge to estimate the window length. Thus, it is more suitable for the application of the field seismic records. The proposed method is no signal attenuation and distortion caused by inappropriate WL.

We use block matching before de-noising firstly. This greatly increases the computational complexity of the algorithm. For block matching, assuming that the size of noisy signal is $N \times N$, the size of block is $1 \times 1$, the step between two blocks is $N s$ and the search window is $N_{h} \times N_{h}$. The time complexity is calculated as

$\frac{N^{2} \times N_{h}^{2}}{N_{s}^{2}}$

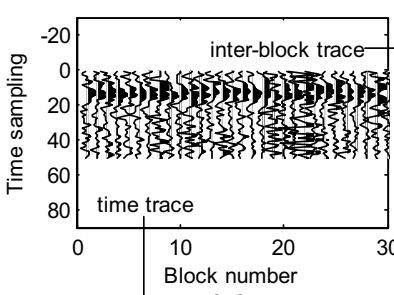

(a)

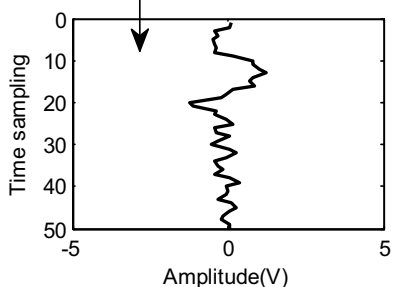

(c)

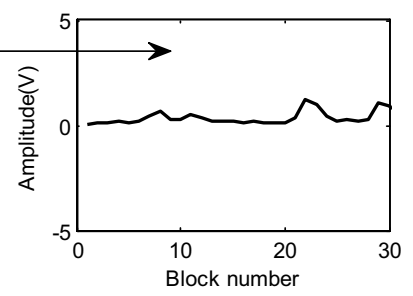

(b)
Fig. 5 a A group of pure signal. b Channel along inter-block direction. $\mathbf{c}$ Channel along time direction 


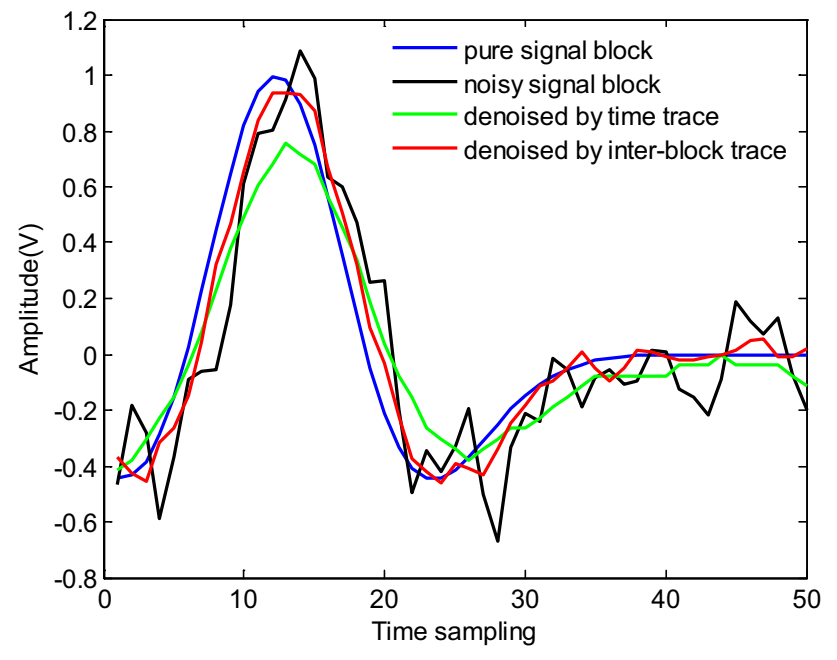

Fig. 6 Result of two filtering methods for block

Our method is able to improve the de-noising performance by sacrificing computational time.

\section{Aggregation}

Through inter-block trace TFPF and block-wise estimates, $\hat{Y}_{x}$ have been obtained. In general, each block is used several times, because it belongs to the different reference block. And, blocks are also overlapping in the original location. This situation leads to overlapping block-wise estimates. To achieve the global estimate $\hat{Y}$, we place block-wise estimates back to original location and propose a computing method:

$\hat{Y}=\frac{\sum_{Y_{R}} \sum_{Z_{R}} \hat{Y}_{x}(a, b)}{c}$

where $a$ and $b$ are coordinates of point in the obtained blockwise estimates. And $c$ is how many times the $\hat{Y}_{x}(a, b)$ has been used.

Above all, implementation and details of AL-TFPF are described at length. Since the AL-TFPF achieves linear enhancement by matching, it does not require dominant frequency of reflection event to estimate the maximum optimal WL. In addition, AL-TFPF is able to avoid the signal loss usually caused by unsatisfactory WLs.

\section{Application of AL-TFPF in broad band signals}

To verify the advantage of AL-TFPF using broad band seismic signals, we simulate seismic signals containing three events, whose dominant frequencies are 25,55 and $85 \mathrm{~Hz}$, respectively. Actual noise is added to the noise-free data and the SNR is $-5.5977 \mathrm{~dB}$. The frequency of noisy signals ranges from 0 to $200 \mathrm{~Hz}$. The results are shown in Figs. 7 and 8 . First, we can find that, after the conventional TFPF $(\mathrm{WL}=13$ ), the result of $85 \mathrm{~Hz}$ has serious distortion of nearly $50 \%$. Then, we can see that there is almost no distortion in the result of conventional TFPF $(\mathrm{WL}=5)$. However, the noise still exists. Finally, after the AL-TFPF, most random noise has been reduced and the reflection events are revealed clearly.

When a signal contains multiple dominant frequencies, the traditional TFPF cannot consider the different dominant frequencies at the same time, so the de-noising effect is poor. However, the AL-TFPF does not need to calculate the window length, so the de-noising effect is better.

\section{Application to the seismic records}

\section{Synthetic seismic record}

To verify the performance of the proposed algorithm, we test our method on a 50-channel synthetic seismic record. The record contains three reflected events with dominant frequencies 45, 35 and $25 \mathrm{~Hz}$, respectively. The sampling frequency is $1000 \mathrm{~Hz}$ and the offset is $30 \mathrm{~m}$. The desired signals are mixed with a series of stochastic shifting in the traces and embedded in real noise with an SNR of $-4.4474 \mathrm{~dB}$. The real noise is intercepted from real seismic

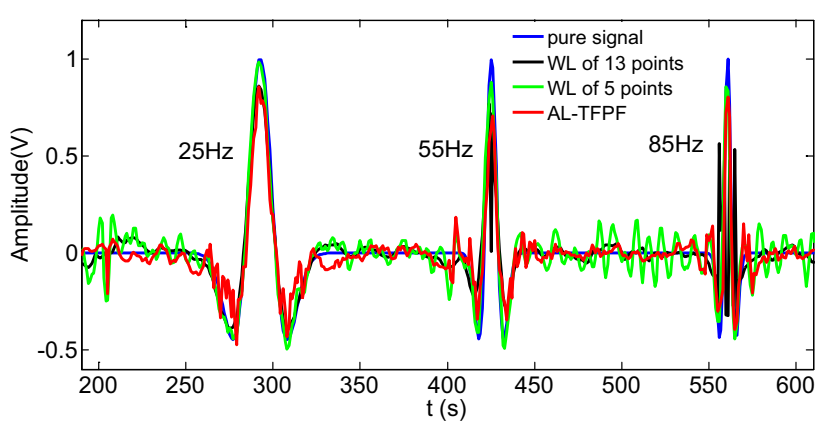

Fig. 7 Results of TFPF with different WLs and AL-TFPFs

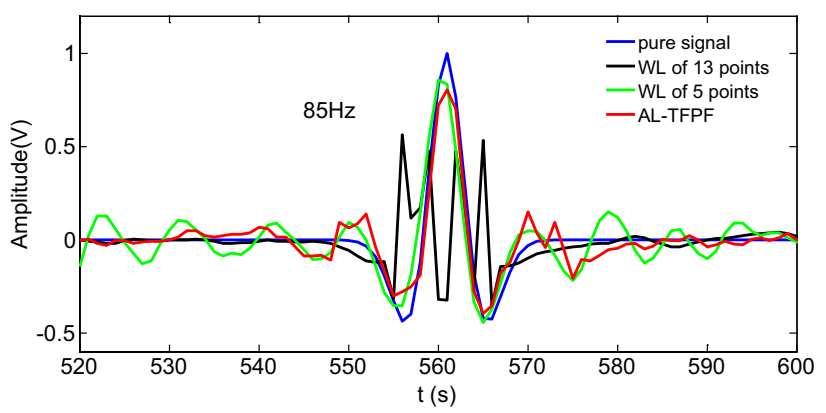

Fig. 8 Enlarged section of $85 \mathrm{~Hz}$

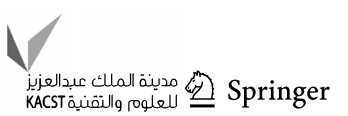


record and the actual noise is a kind of colored noise. The noisy signal is shown in Fig. 9a. We apply the conventional TFPF (WL $=13$ ), the fx-deconvolution (fx-decon), the PTTFPF $(k=0.0001, \mathrm{WL}=25)$ and the AL-TFPF $\left(N_{1}=50\right.$, $\left.N_{p}=4, \tau=150\right)$ to it, respectively. Figure $9 \mathrm{~b}$ shows the denoised result by the conventional TFPF. Figure 9c shows the de-noised result by the fx-decon. Figure $9 \mathrm{~d}$ shows the de-noised result by the PT-TFPF. Figure 9e shows the denoised result by the AL-TFPF. It is obvious that the noisy data are recovered as the desired one, and the random noise is also adequately suppressed through AL-TFPF.

Figure 10 gives the difference between the input data and the de-noised result of each method. Obviously, the AL-TFPF has the least signal leakage. Figure 11 gives the

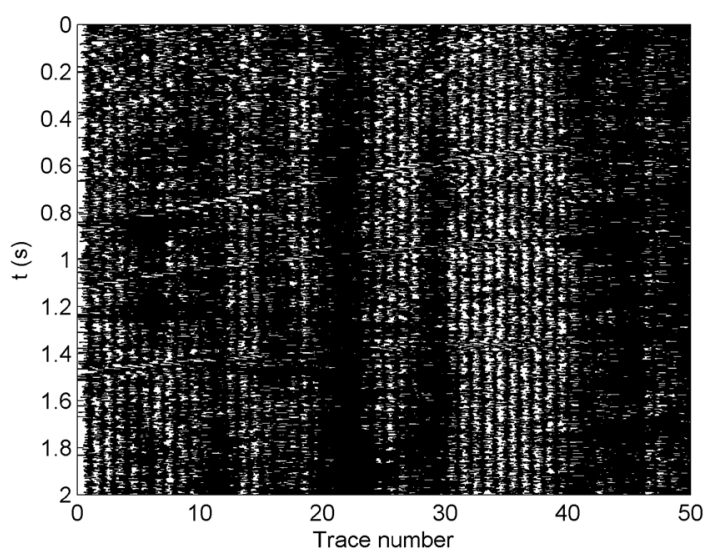

(a)

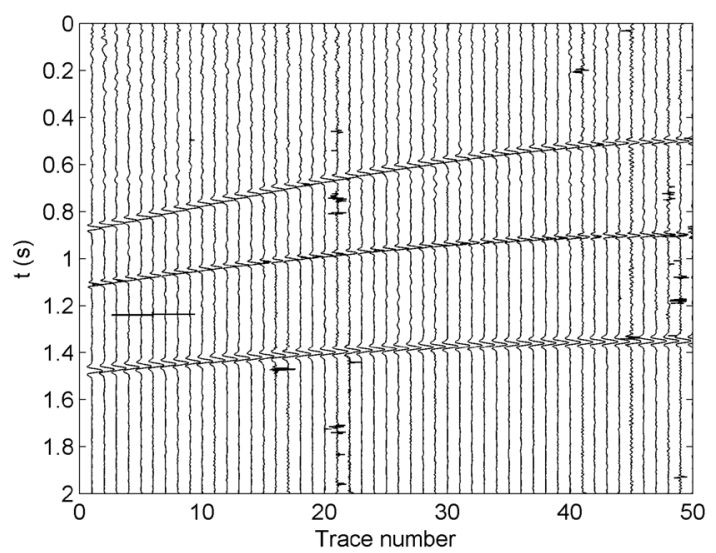

(b)

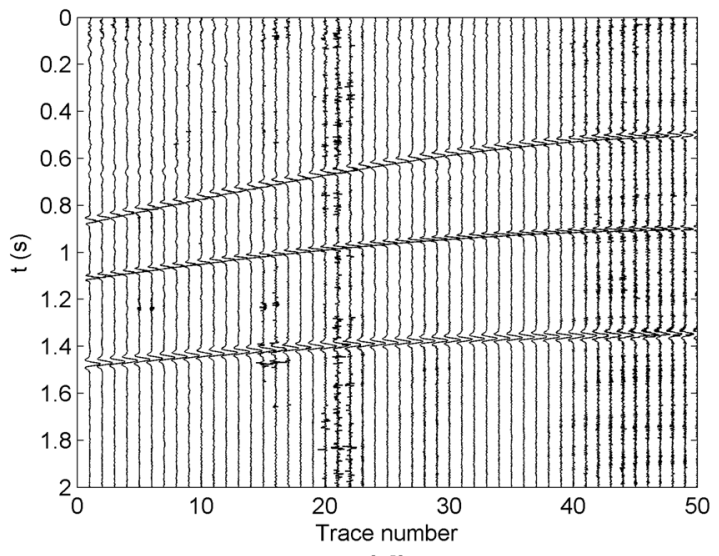

(d)

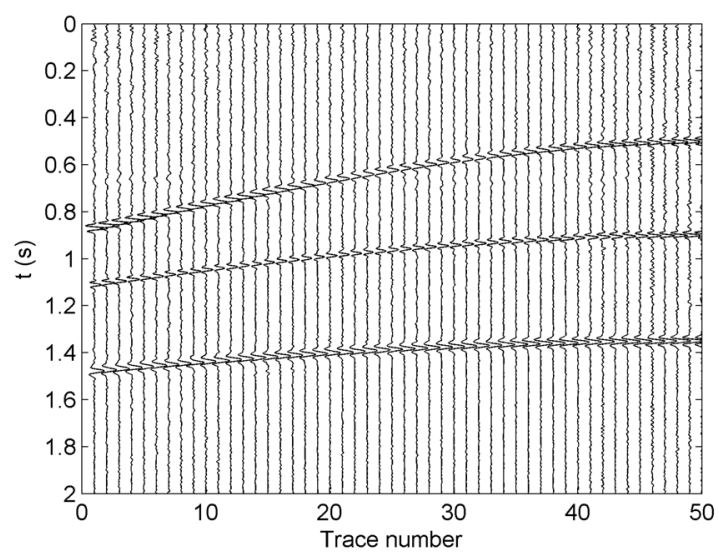

(c)

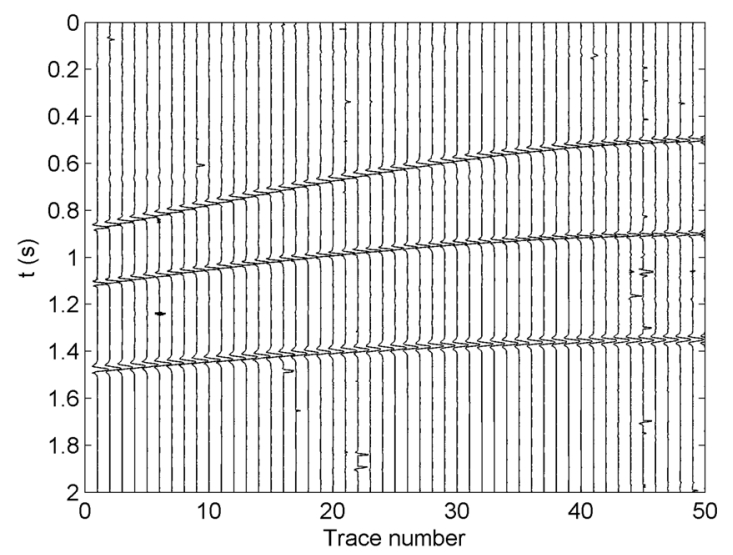

(e)

Fig. 9 a Noisy seismic record. b Result by the conventional TFPF. $\mathbf{c}$ Result by fx-decon. $\mathbf{d}$ Result by PT-TFPF. e Result by AL-TFPF 


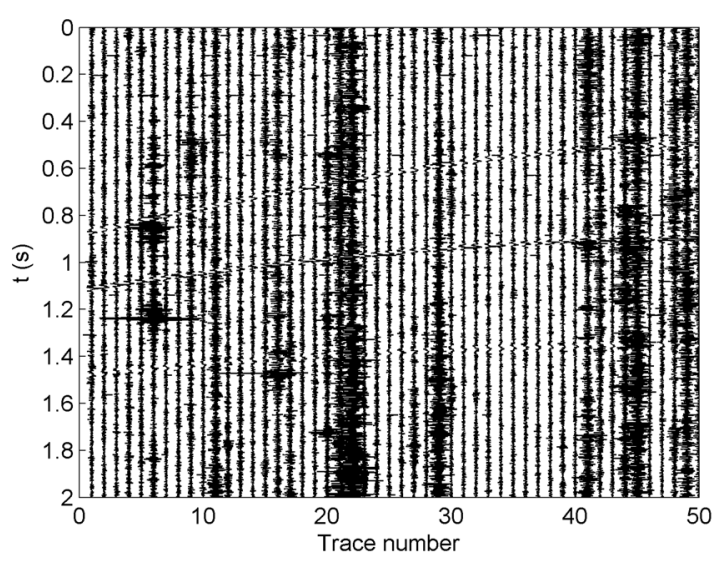

(a)

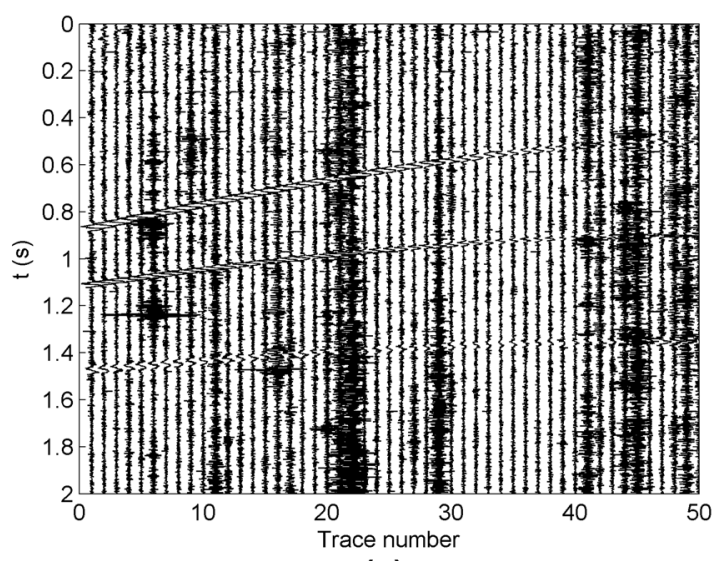

(c)

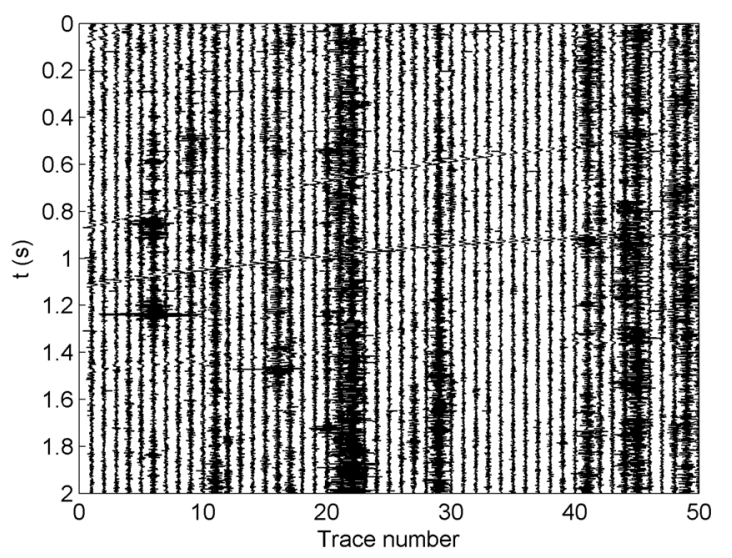

(b)

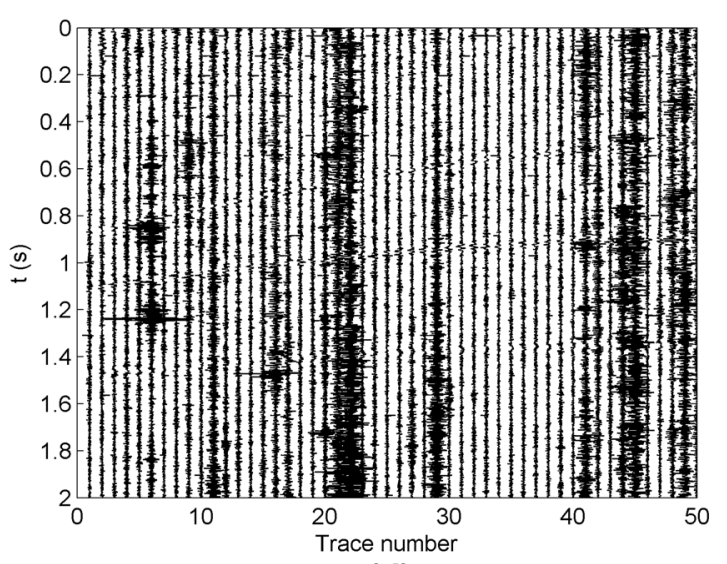

(d)

Fig. 10 a Difference of the conventional TFPF. b Difference of the fx-decon. c Difference of the PT-TFPF. d Difference of the AL-TFPF

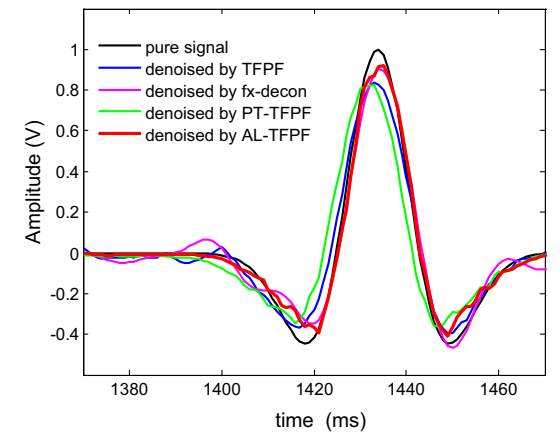

(a)

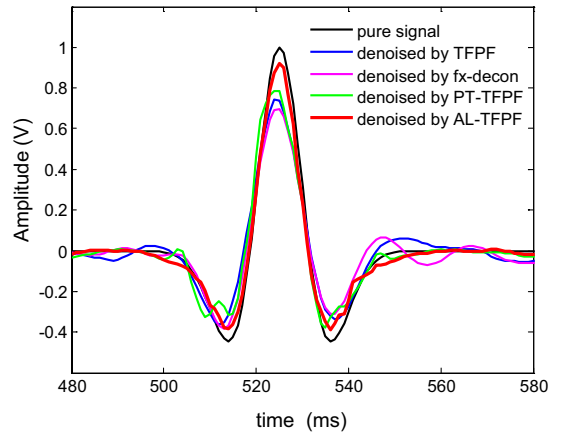

(b)

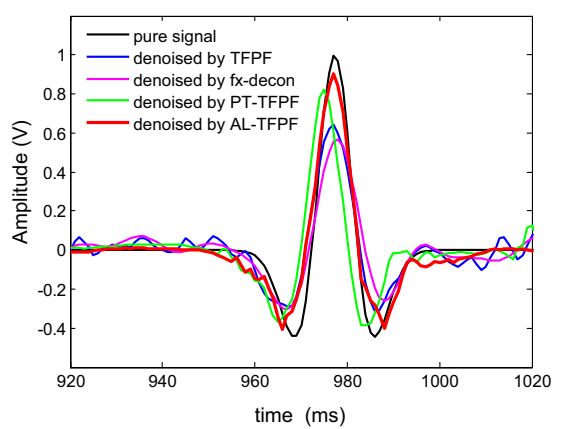

(c)

Fig. 11 a Result of 25-Hz synthetic seismic signal. b Result of 35-Hz synthetic seismic signal. c Result of 45-Hz synthetic seismic signal

detailed comparison of each dominant frequency signal with four methods. The proposed method in this article retains the signal amplitude better for seismic events with different dominant frequencies. Figure 12 gives corresponding spectrum diagram. It can be seen that the energy of the improved method is more stable. For quantitative analysis, the amplitude loss corresponds to these four methods, as shown in Table 1. Bold characters in Table 1 indicate the lowest rate of amplitude loss among the three methods at different frequencies of noisy signals. In terms of amplitude preserving, the fx-decon looses more signals in seismic events with $45 \mathrm{~Hz}$, and the PT-TFPF has been improvement comparing 


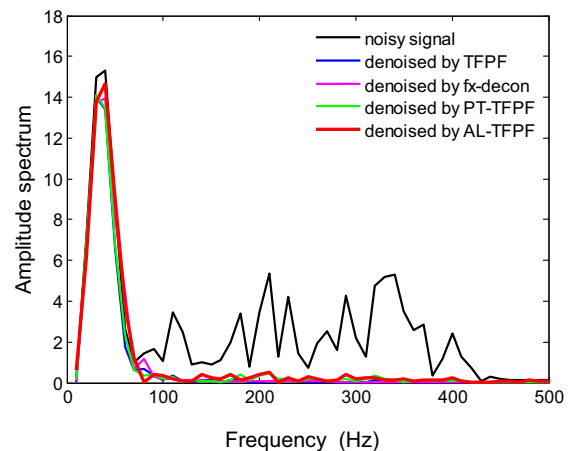

(a)

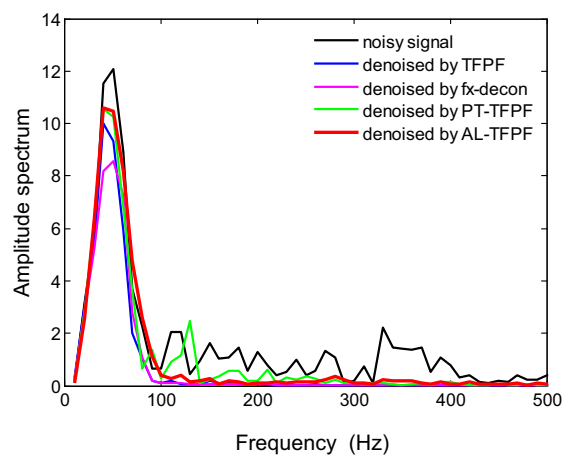

(b)

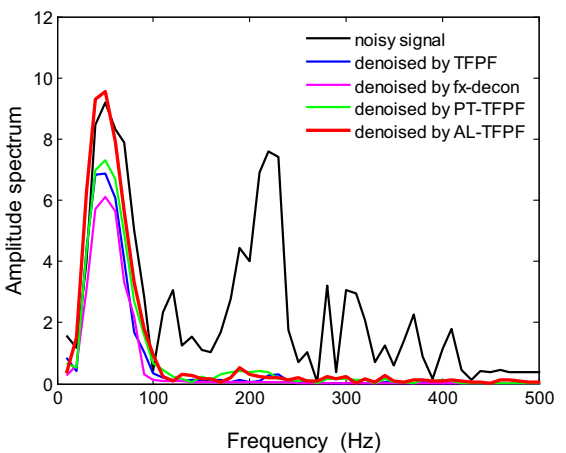

(c)

Fig. 12 a Amplitude spectrum of 25-Hz synthetic signal. b Amplitude spectrum of 35-Hz synthetic signal. c Amplitude spectrum of 45-Hz synthetic signal

Table 1 Amplitude loss with different methods

\begin{tabular}{lccc}
\hline Frequency & $25 \mathrm{~Hz}(\%)$ & $35 \mathrm{~Hz}(\%)$ & $45 \mathrm{~Hz}(\%)$ \\
\hline FX-DECON & 9.60 & 25.43 & 43.26 \\
TFPF & 16.21 & 21.83 & 35.45 \\
PT-TFPF & 17.26 & 12.77 & 17.66 \\
AL-TFPF & $\mathbf{5 . 0 3}$ & $\mathbf{7 . 1 8}$ & $\mathbf{6 . 3 2}$ \\
\hline
\end{tabular}

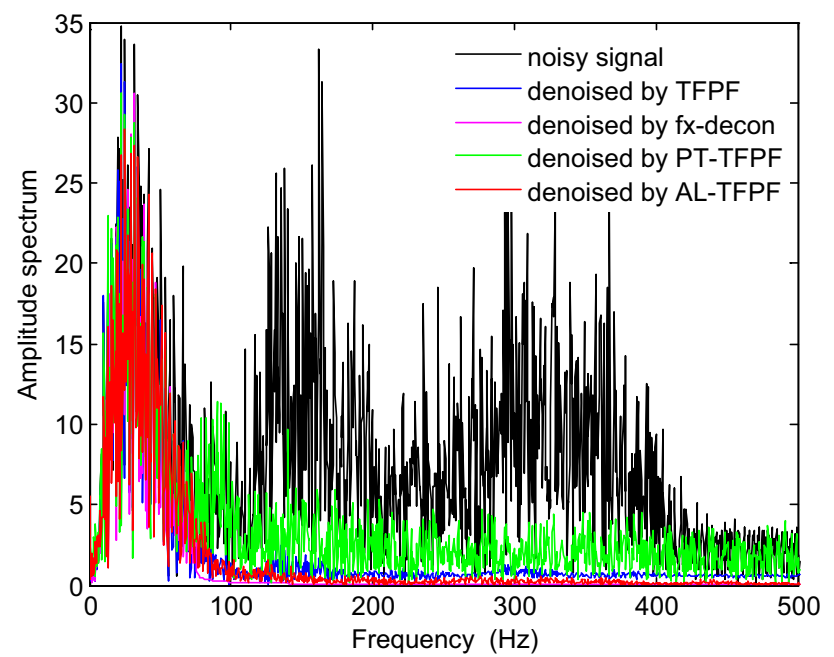

Fig. 13 Amplitude spectrums of single trace with different methods

to TFPF, but AL-TFPF has achieved a better effect. Figure 13 shows spectrums diagram before and after filtering. By comparing with experimental results, a spectrum diagram of ALTFPF more close to of pure signal at below $200 \mathrm{~Hz}$ and over $200 \mathrm{~Hz}$. These results prove that AL-TFPF attenuates the random noise with less loss of the useful components, in particular for high dominant frequency signals.

In order to verify a variety of situations, we adjust the amplitude of noise to obtain different SNRs. The de-noised
Table 2 SNRs with different methods

\begin{tabular}{lrrrrr}
\hline SNR(dB) & 5.3152 & -0.3650 & -4.4474 & -9.9077 & -14.6851 \\
FX- & 11.4671 & 9.0545 & 7.1070 & $\mathbf{4 . 1 8 6 7}$ & $\mathbf{1 . 2 5 4 2}$ \\
DECON & & & & & \\
TFPF & 11.1072 & 8.8778 & 6.3978 & 1.6635 & -2.7759 \\
PT-TFPF & 5.4767 & 4.9483 & 3.9107 & 1.2199 & -2.2759 \\
AL-TFPF & $\mathbf{1 3 . 3 4 0 5}$ & $\mathbf{1 1 . 1 0 2 7}$ & $\mathbf{8 . 2 6 1 2}$ & 3.7192 & 0.5338 \\
\hline
\end{tabular}

SNRs of these four methods are shown in Table 2. Bold characters in the Table 2 indicate the highest SNR of the three methods when the SNR of the noisy signal is different. Comparing with the conventional TFPF and the PT-TFPF, the AL-TFPF gets a higher SNR in a variety of situations. Comparing with the fx-decon, the AL-TFPF suppresses noise better, when SNR is greater than $-9 \mathrm{~dB}$. However, the AL-TFPF's SNR is slightly smaller than the fx-decon's SNR when SNR is less than $-9 \mathrm{~dB}$.

\section{Field data processing}

To test the practicality of the proposed method, we apply the AL-TFPF to a field seismic data acquired from a certain zone in China. Figure 14a shows a common shot point (CSP) record with 168-channel. There are 6000 sample points in each channel. The sampling frequency is $1000 \mathrm{~Hz}$ and the geophone interval is $30 \mathrm{~m}$. In this seismic record, the reflection events are buried in strong random. And there are some areas whose reflection events are too disordered to identify. Moreover, there are some other types of noise, which may be brought in by the collection device.

We apply the conventional TFPF $(\mathrm{WL}=13)$, the PT-TFPF $(k=0.2, \mathrm{WL}=25)$ and the AL-TFPF $\left(N_{1}=100, N_{p}=10\right.$, $\tau=1 \times 10^{-3}$ ) to it, respectively. The amplitude of the actual data is within $10^{-3}$. In order to facilitate later data processing, we set amplitude of the simulation data to 1 . Because 


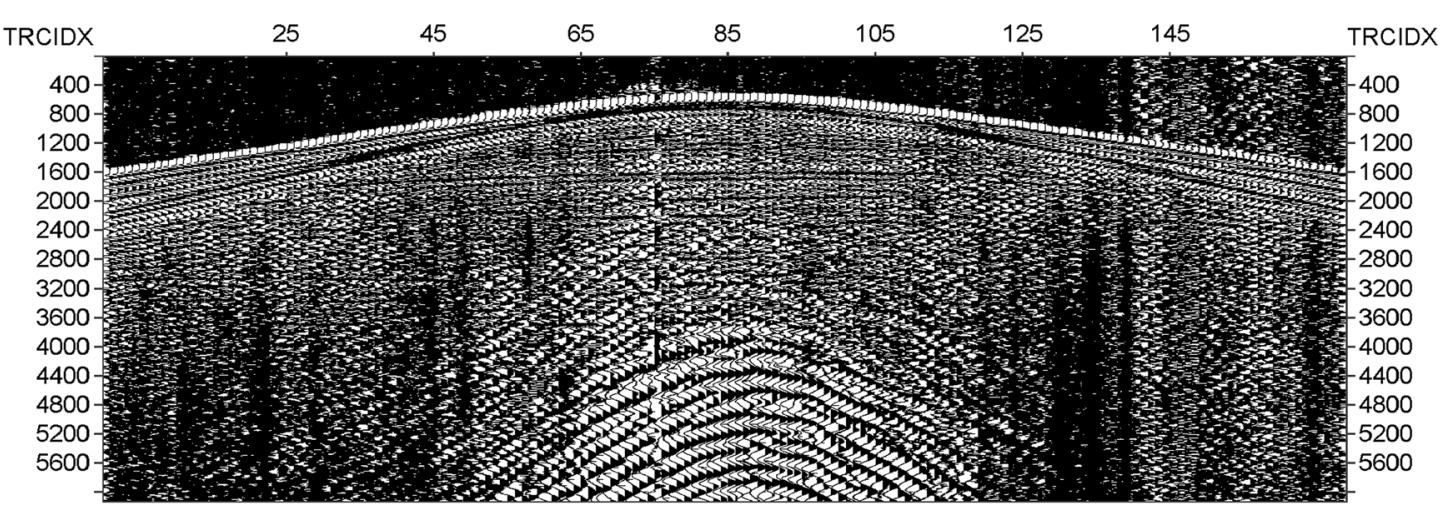

(a)

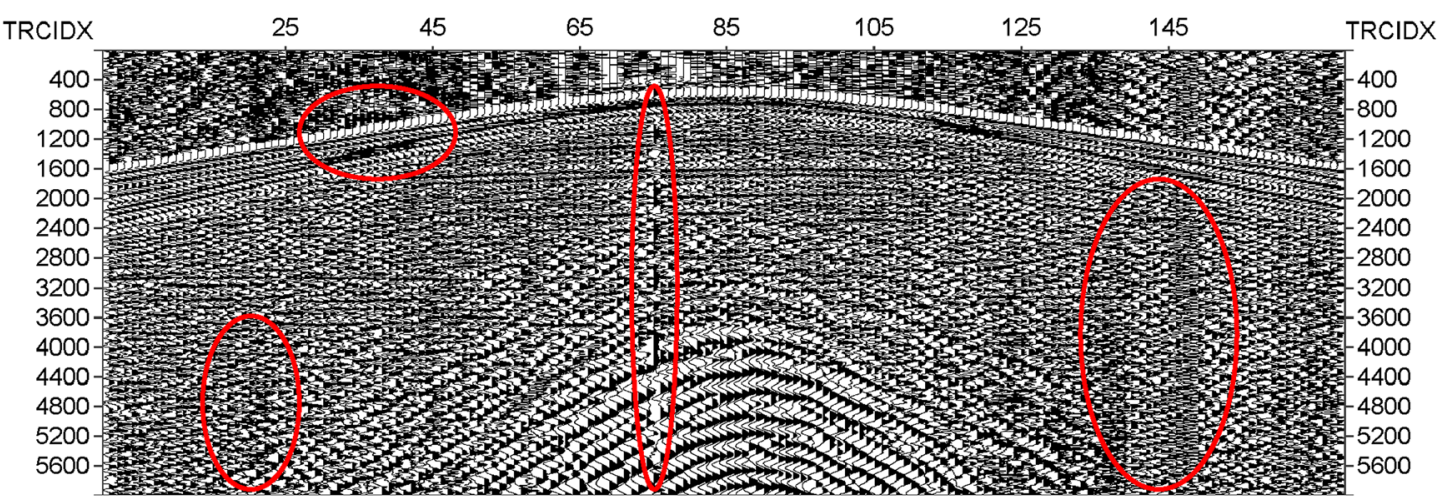

(b)

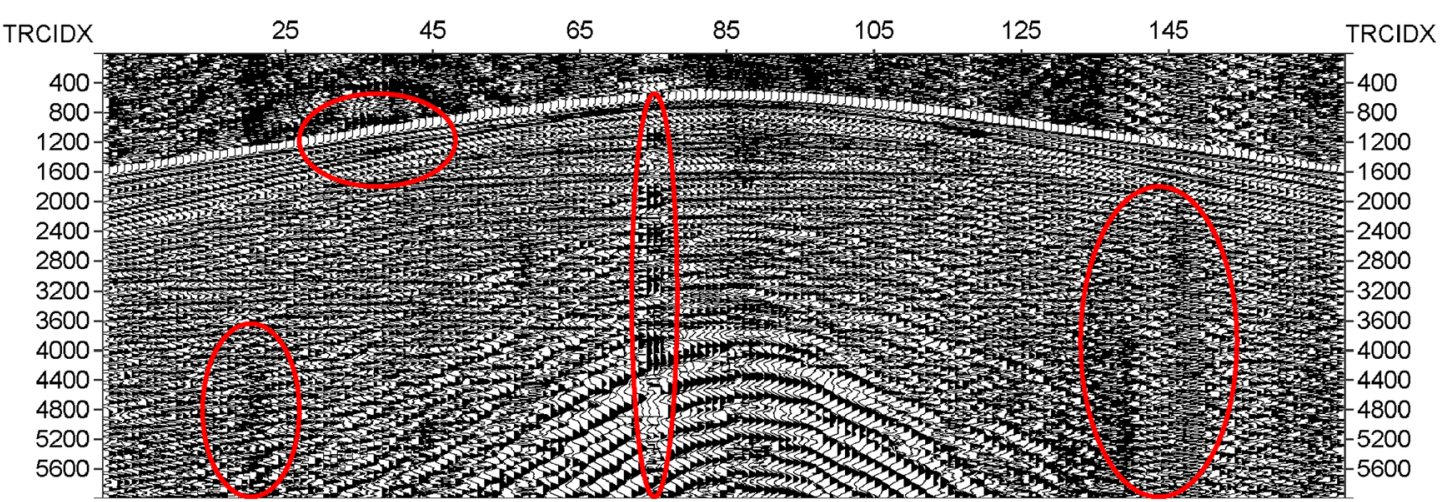

(c)

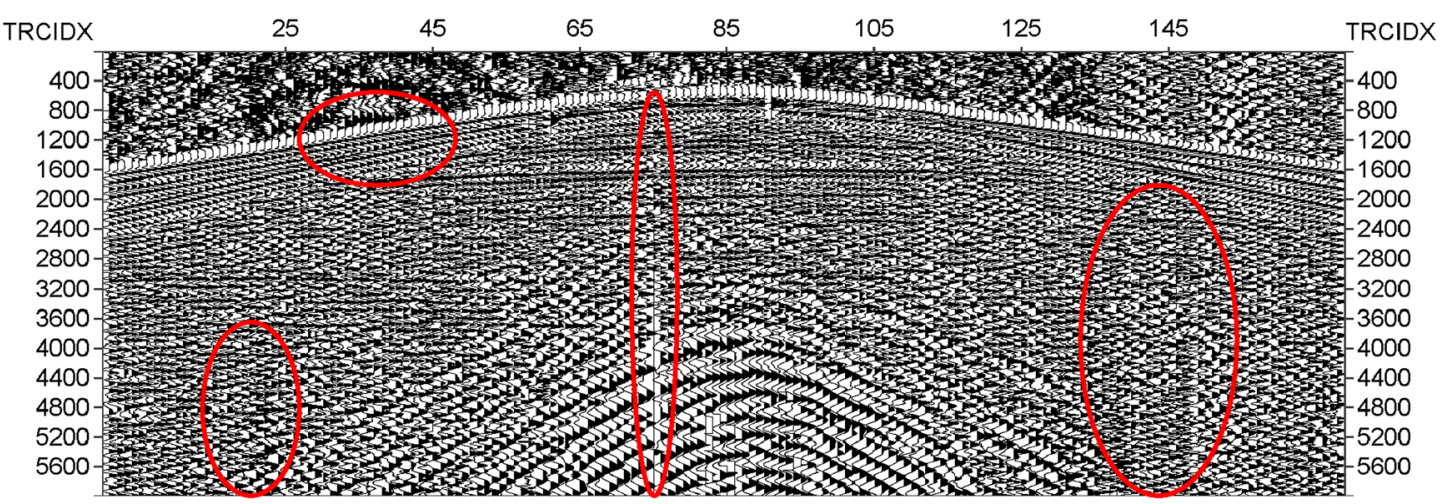

(d)

Fig. 14 a Field seismic record. b Result by the conventional TFPF. c Result by the PT-TFPF. d Result by the AL-TFPF 


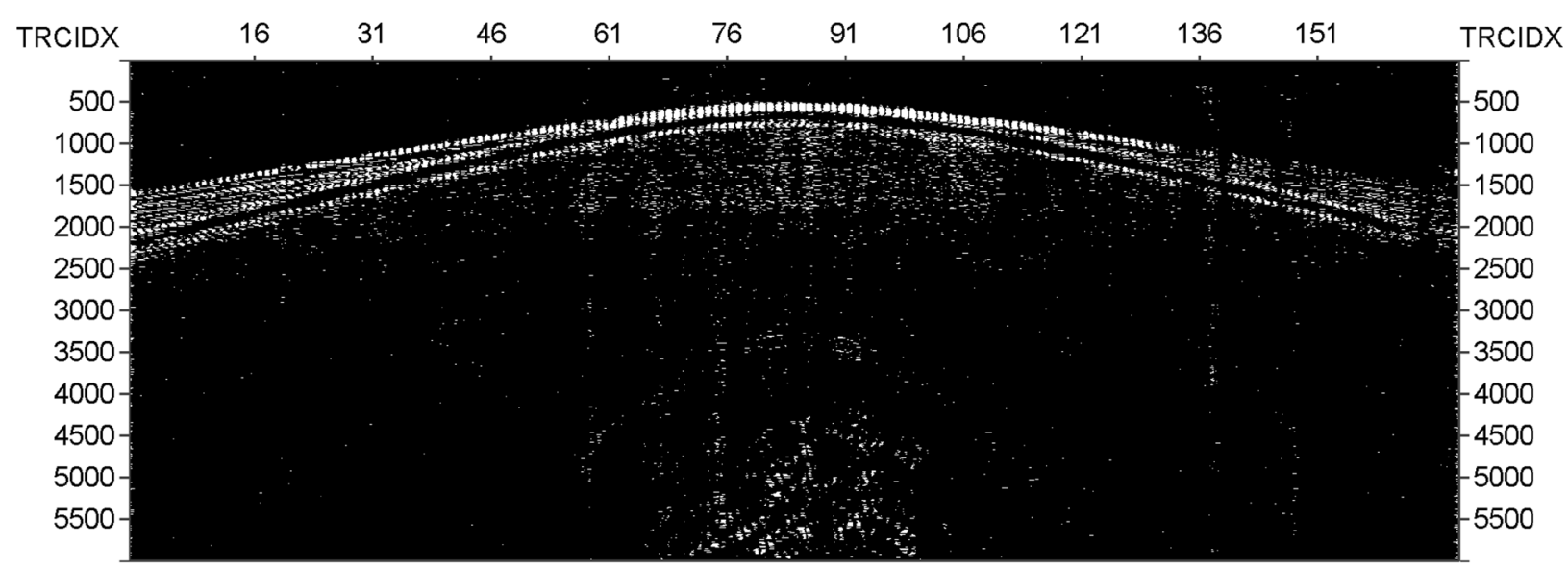

(a)

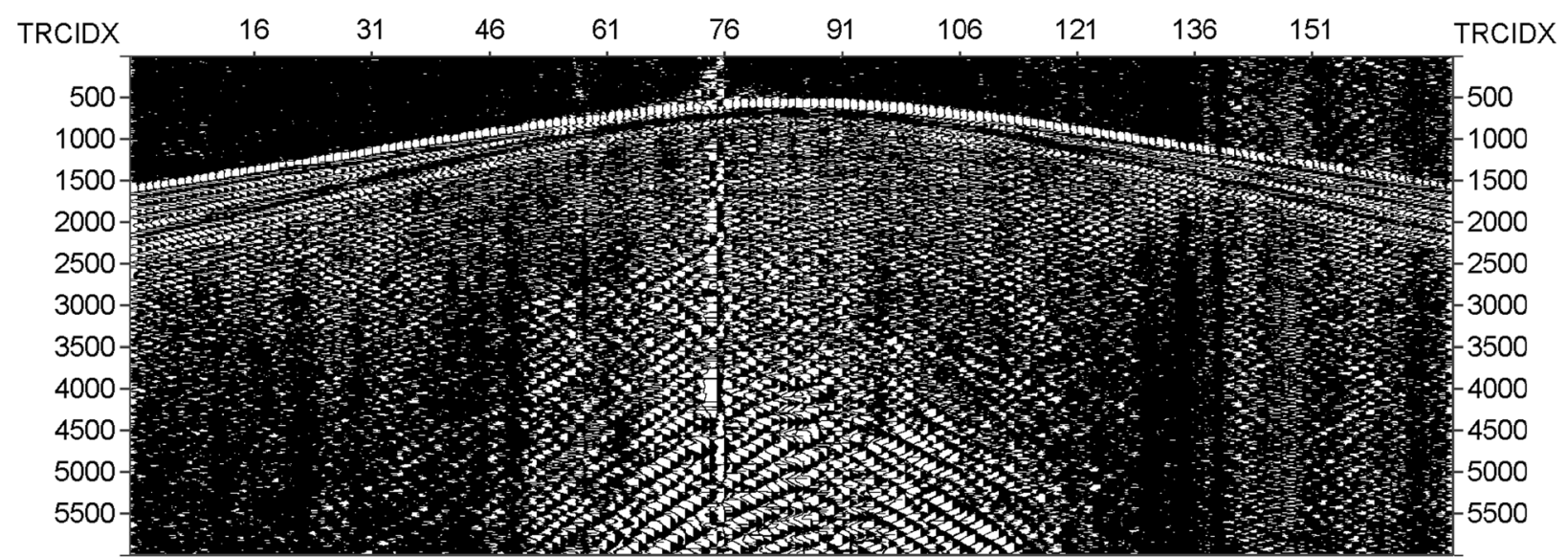

(b)

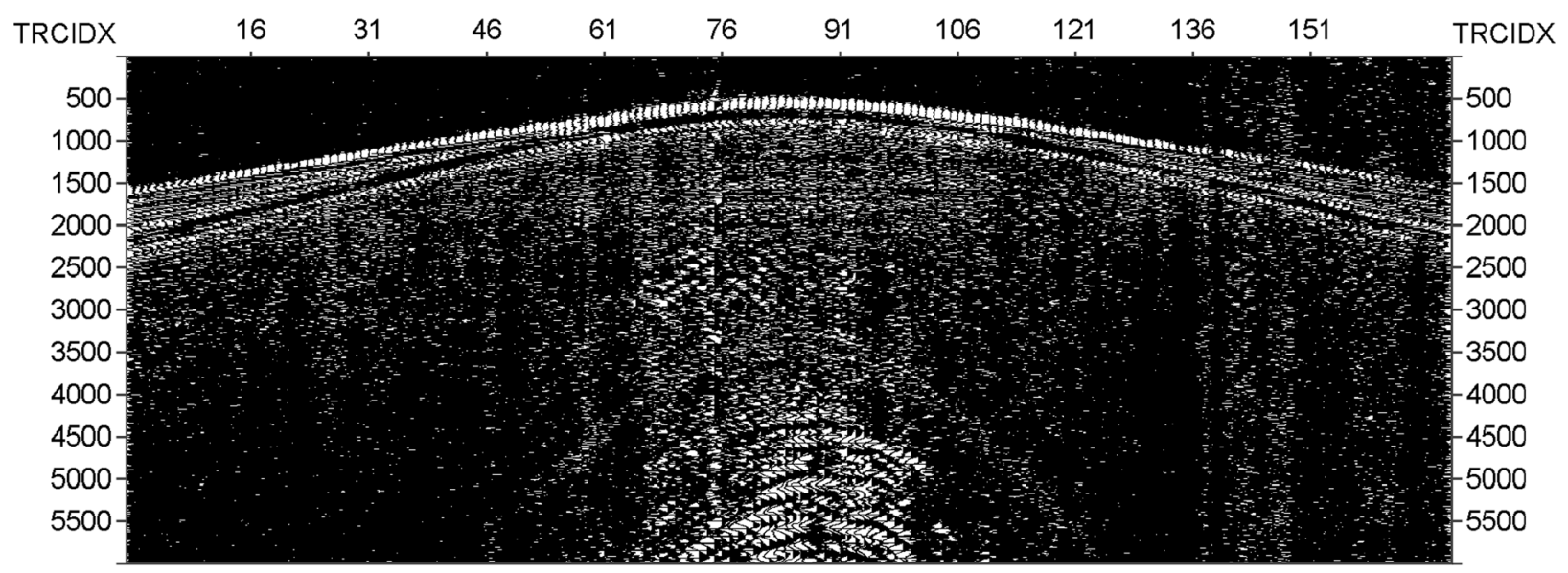

(c)

Fig. 15 a Difference section of the TFPF. b Difference section of the PT-TFPF. c Difference section of the AL-TFPF

the amplitudes are different between the synthetic and the real data, the threshold values are different. The de-noising results after these three methods are given in Fig. 14b-d. We can see that the conventional TFPF performs better in suppressing noise than others. However, background noise still exists, especially in the circled area. Furthermore, the special noise in the middle part of the filed seismic record is not removed. After the PT-TFPF, some originally hidden 
events are revealed. But background noise and special noise also exist. On the contrary, background noise between the reflection events is attenuated more effectively by the ALTFPF, shown as in Fig. 14d. Special noise is also removed. From Fig. 15, we can see that each algorithm has some signal loss. The signal loss of the conventional TFPF is less, but the noise suppression is also less. Moreover, the PT-TFPF algorithm looses the most signal. The AL-TFPF has distinct advantages in both eliminating directional random noise and preserving reflected signals in comparison with many other methods.

\section{Conclusion}

The AL-TFPF seismic de-noising method is proposed in this article. This novel method resamples the noisy seismic record through matching based on spatial correlation between adjacent channels. Through this step, the ALTFPF searches the events adaptively to resolve the problem caused by fixed form filtering trace. Therefore, the AL-TFPF is more suitable for the field record with complex events. In addition, the number of the resampling points is similar along inter-block trace in a group. That is to say, linearity is implemented and enhanced by inter-block filtering trace instead of window. This method resolves not only the problem of window length selection, but also signal attenuation even distortion.

By the comparison with the conventional TFPF, fx-decon and the PT-TFPF, we deduced that AL-TFPF performs better in effective components preservation, in particular for high dominant frequency reflection events. Moreover, the recovered events became clearer and more continuous.

Finally, due to the influence of noise, the linearity of the effective signals cannot be improved completely by weighted Euclidean distance in noisy seismic record. The AL-TFPF also needs further research.

Acknowledgements The authors would like to thank the National Natural Science Foundation of China (4157 4096), and the key Science Foundation of the Department of Science and Technology of Jilin Province (20180201081SF)

Funding These funders include the key project of National Natural Science Foundation of China (Grant No. 41574096), the key Science Foundation of the Department of Science and Technology of Jilin Province (Grant No. 20180201081SF), Jilin Provincial Special Funding for Industrial Innovation (Grant No. 2017C031-1) and Jilin UniversityProvince Collaboration Funding (Grant No. SXGJQY2017-9).

Open Access This article is distributed under the terms of the Creative Commons Attribution 4.0 International License (http://creativeco mmons.org/licenses/by/4.0/), which permits unrestricted use, distribution, and reproduction in any medium, provided you give appropriate credit to the original author(s) and the source, provide a link to the Creative Commons license, and indicate if changes were made.

\section{References}

Abma R, Claerbout J (1995) Lateral prediction for noise attenuation by $t-x$ and $f-x$ techniques. Geophysics 60:1887-1896. https:// doi.org/10.1190/1.1443920

Boashash B, Mesbah M (2004) Signal enhancement by time-frequency peak filtering. IEEE Trans Signal Process 52:929-937. https://doi. org/10.1109/TSP.2004.823510

Chen YK, Ma JT (2014) Random noise attenuation by $f-x$ empiricalmode decomposition predictive filtering. Geophysics 79:V81V91. https://doi.org/10.1190/GEO2013-0080.1

Górszczyk A, Adamczyk A, Malinowski M (2014) Application of curvelet denoising to $2 \mathrm{D}$ and $3 \mathrm{D}$ seismic data-practical considerations. J Appl Geophys 105:78-94. https://doi.org/10.1016/j.jappg eo.2014.03.009

Lin HB, Li Y, Yang BJ (2007) Recovery of seismic events by time frequency peak filtering. IEEE Int Conf Image Process 5:2693-2696. https://doi.org/10.1109/ICIP.2007.4379860

Lin HB, Li Y, Yang BJ (2008) Varying-window-length time-frequency peak filtering and its application to seismic data. Comput Intell Secur. https://doi.org/10.1109/cis.2008.160

Lin HB, Li Y, Xu XC (2011) Segmenting time-frequency peak filtering method to attenuation of seismic random noise. Chin J Geophys 54:1358-1366 (in Chinese)

Lin HB, Li Y, Ma HY et al (2015) Adaptive fission particle filter for seismic random noise attenuation. IEEE Geosci Remote Sens Lett 12:1918-1922. https://doi.org/10.1109/LGRS.2015.2438229

Liu B, Sacchi M (2004) Minimum weighted norm interpolation of seismic records. Geophysics 69:1560-1568. https://doi. org/10.1190/1.1836829

Liu CM, Wang DL, Wang T et al (2014a) Random seismic noise attenuation based on the Shearlet transform. Acta Pet Sin 35:692-699. https://doi.org/10.7623/syxb201404009

Liu SC, Gao EG, Xun C (2014b) Seismic data denoising simulation research based on wavelet transform. Appl Mech Mater V490 V491:1356-1360. https://doi.org/10.4028/www.scientific.net/ AMM.490-491.1356

Tian Y, Li Y (2014) Parabolic-trace time-frequency peak filtering for seismic random noise attenuation. IEEE Geosci Remote Sens Lett 11:158-162. https://doi.org/10.1109/LGRS.2013.2250906

Tian YN, Li Y, Yang BJ (2014) Variable-eccentricity hyperbolic-trace TFPF for seismic random noise attenuation. IEEE Geosci Remote Sens 52:6449-6458. https://doi.org/10.1109/TGRS.2013.2296603

Trad D, Ulrych T, Sacchi M (2002) Accurate interpolation with high resolution time-variant Radon transforms. Geophysics 67:644656. https://doi.org/10.1190/1.1468626

Wang CT, Wei W, Liu YH (2010) Seismic data noise suppression technique based on independent component analysis. Comput Simul 27:253-257

Wu N, Li Y, Yang BJ (2011) Noise attenuation for 2-D seismic data by radial-trace time-frequency peak filtering. IEEE Geosci Remote Sens Lett 8:874-878. https://doi.org/10.1109/LGRS.2011.21295 52

Publisher's note Springer Nature remains neutral with regard to jurisdictional claims in published maps and institutional affiliations. 